\title{
Ginseng Total Saponin Attenuate Cardiac Hypertrophy Induced by Homocysteine in Rats
}

\author{
Jong-Hoon Kim* \\ Department of Veterinary Physiology, College of Veterinary Medicine, Chonbuk National University, \\ Biosafety Research Institute, 664-14, 1GA, Duckjin-Dong, Duckjin-Gu, Jeonju City, Jeollabuk-Do 561-756, Korea
}

(Received November 18, 2009; Revised December 3, 2009; Accepted December 9, 2009)

\begin{abstract}
Recent studies have shown that Panax ginseng has a variety of beneficial effects on the cardiovascular system. Homocysteine (Hcy), which is derived from methionine, has been closely associated with the increased risk of cardiovascular diseases. In the present study, whether the in-vivo long-term co-administration of ginseng total saponins (GTS), active ingredients of Panax ginseng, with $L$-methionine (Met) inhibits methionine-induced hyperhomocysteine (HHcy) and H-Hcy-induced cardiovascular dysfunctions was investigated, and it was found that the plasma Hcy level, which was measured after 30 and 60 days, in the GTS+Met co-administration group was more significantly reduced than in the Metalone-treatment group. The left-ventricle (LV) wall thickness of the heart was likewise examined in each treatment group, and it was found that the co-administration of GTS with Met significantly reduced the Met-induced LV wall thickness. The results of the study indicate that the in-vivo long-term co-administration of GTS with Met not only inhibited H-Hcy induced by long-term Met-alone administration but also attenuated the H-Hcy-induced cardiovascular dysfunctions in rats.
\end{abstract}

Key words : ginseng total saponin, homocysteine, cardiac hypertrophy, echocardiograhy, left ventricle thickness

\section{INTRODUCTION}

Homocysteine (Hcy) is a thiol-containing amino acid derived from $L$-methionine (Met), one of essential amino acids. Hcy is produced entirely from the methylation cycle, since it is totally absent from any dietary source. ${ }^{1,2)}$ Three forms of Hcy are usually present in plasma, whereby most of it $(80-90 \%)$ is bound to proteins; a smaller part is available as free amino acid, and traces are present as disulphide forms. Studies in animal models and humans have demonstrated that hyperhomocysteinaemia (H-Hcy) first induces endothelial dysfunctions. ${ }^{3-11)}$ Endothelium dysfunctions are known as an initial step of arteriosclerosis and induces alterations of blood vessel contraction and relaxation, permeability, and changing platelet and leukocyte adhesions. Although the molecular mechanisms by which H-Hcy impairs the blood vessel wall and promotes arteriosclerosis are still not clearly elucidated, it is believed that a high concentration of plasma Hcy is the main causes of the vascular endothelium injury, ${ }^{12,13)}$ and resulting in increased oxidative stress and decreased nitric oxide (NO) bioavailabil-

* Corresponding author. E-mail: jhkim1@ chonbuk.ac.kr Phone: +82-63-270-2563, Fax: +82-63-270-3780 ity. ${ }^{14,15)}$ On the other hand, H-Hcy can also affect left ventricular (LV) hypertrophy and cardiac fibrosis in animal models that is reversed with folate treatment. ${ }^{16,17)}$

Ginseng, a widely recognized herbal drug, has been reported to have a wide range of therapeutic and pharmacological uses. Ginseng's genus name Panax is derived from the Greek words pan (all) and akos (cure), meaning cure-all. Ginseng root has been used extensively in Korean and Chinese medicines and has become increasingly popular in the Western world for its alleged tonic effect and possible preventive and restorative properties. There are increased experimental evidences concerning the beneficial effects of ginseng roots in cardiovascular abnormalities. These studies have shown that ginsenosides total saponins (GTS), the major constituents of ginseng, have protective effects from vascular injuries due to various causes. ${ }^{18,19)}$ For example, it has been reported to be effective in treating cardiovascular diseases such as coronary thrombosis and in improving cardiac muscle microcirculation. Administration of GTS improved myocardial relaxation function, ${ }^{20)}$ lowered cerebrovascular resistance, suppressed proliferation of aortic smooth muscle cells induced by hypercholesterolemic serum, ameliorated cardiac function in the early stage postburn, ${ }^{21)}$ and 
finally enhanced fibrinolytic activity of bovine aortic endothelial cells. ${ }^{22)}$ Those effects provide one possibility that GTS might be also a valuable approach for the protection to the injured blood vessels by H-Hcy. However, no previous work has examined whether GTS attenuates $\mathrm{H}-\mathrm{Hcy}$ and has protective effects against cardiovascular dysfunctions after H-Hcy induced by in vivo long-term administration of Met. Here, we investigated whether the long-term co-administration of GTS with Met could attenuate $\mathrm{H}-\mathrm{Hcy}$ induced by in vivo long-term Met alone treatment in rats and also examined whether the long-term treatment of GTS with Met could ameliorate cardiovascular dysfunctions such as LV hypertrophy induced by $\mathrm{H}-$ $\mathrm{Hcy},{ }^{23)}$ We found that co-administration of GTS with Met not only reduced Met-induced H-Hcy but also attenuated LV wall thickness. These results indicate that GTS might be useful agent in attenuating Met-induced H-Hcy as well as H-Hcy-induced cardiovascular abnormalities.

\section{METERIALS AND METHOSD}

\section{Animals}

Forty male Wistar rats (weighing $\approx 150 \mathrm{~g}$; Charles River, KFT) were housed with free access to commercial food pellets (LSM, Bacutil, Poland), as well as tap water $a d$ libitum. All the animals were kept in light-dark conditions $\mathrm{L}: \mathrm{D}=12: 12$. Light was turned on at 07:00. Animal care and handling was in accordance with the highest standards of institutional guidelines. The animals were divided into four groups with ten rats. Group 1: Normal control group with only tap water. Group 2: Met and succinylsulfathiazole (SST) administration in the tap water for a period of 30 and 60 days. Group 3: GTS alone treatment group for a period of 30 and 60 days. Group 4: GTS+Met+SST co-treatment group for a period of 30 and 60 days. SST was used to avoid bacterial proliferation and subsequent folate production. ${ }^{24,25,26)}$

\section{Drug Administrations}

Fig. 1 shows the chemical structures of ginsenosides. GTS was kindly provided by the Korea Ginseng Corporation (Daejon, South Korea). GTS dissolved in saline was administrated intraperitoneally (i.p.) to rats at a dose (50 $\mathrm{mg} / \mathrm{kg}$, twice/day, every $12 \mathrm{~h}$ interval for 30 and 60 days, $\mathrm{n}=10$, each group). GTS administration began on day 0 , followed by Met on day 3. Met was administrated via oral (p.o.) route to rats. Met-caused H-Hcy was induced by administration of the dissolved Met $(1.0 \mathrm{~g} / \mathrm{kg}$ body weight/day) and SST (0.5 g/kg body weight/day) in the

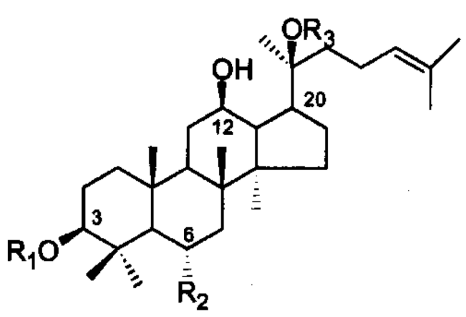

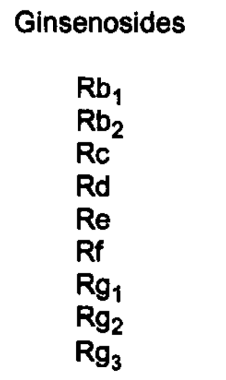

$R_{1}$
$-G l c_{2}-G l c$
$-G l c_{2}-G l c$
$-G l c_{2}-G l c$
$-G l c_{2}-G l c$
$-H$
$-H$
$-H$
$-H$
$-G \mid c_{2}-G l c$
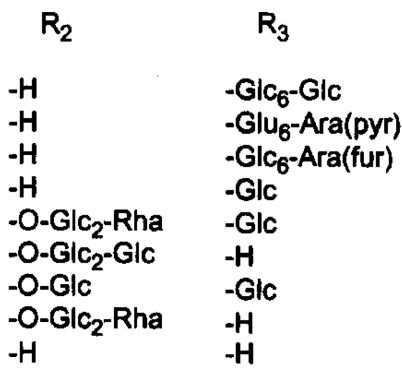

Fig. 1. Structures of the nine representative ginsenosides. They differ at three side chains attached the common steroid ring. Abbreviations for carbohydrates are as follows: Glc, glucopyranoside; Ara (pyr), arabinopyranoside; Rha, rhamnopyranoside. Superscripts indicate the carbon in the glucose ring that links the two carbohydrates.

tap water for a period of 30 and 60 days. The dosage of Met and SST administered per animal was based on average daily fluid intake and SST was used to avoid bacterial proliferation and subsequent folate production. ${ }^{27-29)}$ Control vehicle group was administered only with tap water. Animals of each group were housed separately, and fed standard rat chow, and were weighed at 30 and 60 days, respectively.

\section{Determination of Serum Hcy}

Blood was collected from the femoral artery of fasting rats. It was immediately cooled on ice and centrifuged at $3,000 \times g$ for $20 \mathrm{~min}$ at $4^{\circ} \mathrm{C}$ to limit the release of Hcy from blood cells. Serum was then stored at $-20^{\circ} \mathrm{C}$ until assayed. Total Hcy concentrations were measured by a high-performance liquid chromatography (HPLC) technique with fluorometric detection according to Ulbink et al. ${ }^{30}$ ) Briefly, $240 \mu \mathrm{l}$ of serum and $60 \mu \mathrm{l}$ of internal standard (Nacetyl-L-cysteine, $50 \mu M$ final concentration) was reduced for 30 minutes at $4^{\circ} \mathrm{C}$ with $30 \mu \mathrm{l}$ of tri-n-butyl phosphine $(10 \%)$. Deproteinization was performed with $300 \mu \mathrm{l}$ of $10 \%$ trichloroacetic acid. After centrifugation, $100 \mathrm{ml}$ of the clear supernatant was mixed with $20 \mu \mathrm{l}$ of $1.55 \mathrm{M}$ $\mathrm{NaOH}, 250 \mu \mathrm{l}$ of $0.125 M$ borate buffer (pH 9.5), and 50 $\mu \mathrm{L}$ of $1 \mathrm{mg} / \mathrm{ml}$ 7-fluorobenzo-2-oxa-1,3-diazole-4-sulfonate. After derivatization at $60^{\circ} \mathrm{C}(1 \mathrm{~h})$, the sample was ana- 
lyzed by HPLC (JASCO International Co Ltd), equipped with a fluorescence detector (LC 1255; GBC Scientific Equipment Pty Ltd). Separation was carried out on a $2003 \times 4.6 \mathrm{~mm} \times 5 \mu M$ Nucleosil C18 column. The eluant was $0.1 \mathrm{M}$ acetate buffer ( $\mathrm{pH} 4.0$ ) containing $2 \%$ methanol. The fluorescence intensities were measured with excitation at $386 \mathrm{~nm}$ and emission at $516 \mathrm{~nm}$.

\section{Measurement of Blood Pressure}

Arterial pressure (AP) was continuously recorded in conscious unrestrained rats. ${ }^{31)}$ Briefly, the rats were anesthetized with a mixture of ketamine $(100 \mathrm{mg} / \mathrm{kg}$, i.p. $)$ and acepromazine $(5 \mathrm{mg} / \mathrm{kg}$, i.p.). A polyethylene catheter (PE10 connected to PE-50) was chronically placed into the lower abdominal aorta via the left femoral artery for measurement of AP. The catheter was tunneled subcutaneously and exteriorized through the interscapular skin. After 2 days of recovery, the aortic catheter was connected to a pressure transducer via a rotating swivel that allowed the rat to move freely in the cage. After approximately $3 \mathrm{~h}$ of habituation, the AP signal was digitized by a microcomputer for $2 \mathrm{~h}(12.00-14.00 \mathrm{~h})$. Systolic AP and diastolic AP values were determined on-line. Using offline analysis, the mean values of these parameters were calculated for a period of $2 \mathrm{~h}$ and served as systolic AP and diastolic AP. The standard deviation of the pressure during a $2 \mathrm{~h}$ interval was calculated and defined as the quantitative parameter of blood pressure.

\section{Echocardiography}

Transthoracic $M$-mode and Doppler echocardiography were performed at 60 days after co-administration of GTS and Met, using an ultrasonographic system (SSD-5500, Aloka, Tokyo, Japan) with a $10-\mathrm{MHz}$ transducer. Rats were sedated with intraperitoneal ketamine $\mathrm{HCl}(50 \mathrm{mg} /$ $\mathrm{kg})$ and xylazine $\mathrm{HCl}(5 \mathrm{mg} / \mathrm{kg})$. Standard techniques were used to obtain M-mode echocardiograms from short-axis views at the papillary muscle level of the LV. LV posterior wall thickness at end-systole and diastole (LVPWTs, LVPWTd) were measured. End-diastole and end-systole were defined at the time of the most posterior and anterior excursion of LVPW respectively. Left longitudinal imaging was performed at approximately $45^{\circ}$ through the left parasternal rib space with a maximum imaging depth of $40 \mathrm{~mm}$. M-mode echocardiography was recorded at the level of the mitral valves at a paper speed of $100 \mathrm{~mm} / \mathrm{s}$. LVPWTs and LVPWTd were measured by the leadingedge method from at least three consecutive cardiac cycles on the $M$-mode tracing as proposed by the Ameri- can Society for Echocardiography. ${ }^{32)}$

\section{Data analysis}

All data are reported as means \pm SEM. Statistical analysis was performed with two-tailed Student's unpaired $t$ test, except for the vascular reactivity study, for which two-way ANOVA for repeated measures was used to compare two curves. The threshold for statistical significance was $p<0.05$.

\section{RESULTS AND DISCUSSION}

\section{Body weight.}

We have measured the body weight, food, and water intake during the long-term administration of normal control, GTS alone, Met alone, or GTS+Met group. The initial body weight of normal control, GTS alone, Met alone, or GTS+Met group was 138.5 $\pm 9.6,140.7 \pm 10.5,142.5 \pm 8.8$, $139.7 \pm 8.2 \mathrm{~g}$, respectively. The body weight in normal control, GTS, Met, and GTS + Met group after 30 and 60 days was not significantly different among them. Thus, the body weight of normal control, GTS, Met, and GTS + Met group was $387.6 \pm 18.3,377.4 \pm 22.8,386.5 \pm 25.1$ and $379.7 \pm 24.1 \mathrm{~g}$ after 30 days, respectively and $482.3 \pm 28.3$, $481.5 \pm 32.7,488.9 \pm 35.0$, and $479.8 \pm 29.7$ after 60 days, respectively. No significant changes were also observed for initial plasma Hcy level in all four groups before Met administration (data not shown). During whole experimental periods, the amounts of water and food intakes in each group were not significantly different among four groups (data not shown).

Effects of blood pressure, heart rate, pulse pressure and mean pressure

We next measured changes of blood pressure, heart rate (HR), pulse pressure (PP) and mean pressure in each group. There was no significantly difference in systolic pressure (SP), diastolic pressure (DP) and HR among groups (Table 1). Other hemodynamic parameters such as $\mathrm{PP}$, mean AP also did not significantly differ among all four groups (Table 1).

\section{Effects of GTS on plasma Hcy concentration}

We measured plasma Hcy levels in each administration group. Plasma Hcy levels between normal control and GTS alone group after 30 and 60 days were not significantly different from each other. Con : GTS $=8.8 \pm 2.9$ : $7.3 \pm 2.5 \mathrm{mM}$ after 30 days and $5.4 \pm 3.1$ and $7.3 \pm 3.2 \mathrm{mM}$ after 60 days, respectively. Plasma Hcy levels were sig- 
Table 1. Hemodynamics after 30 and 60 days of treatment with methionine and GTS

\begin{tabular}{ccccccccc}
\hline \hline & \multicolumn{4}{c}{ After 30 days } & \multicolumn{3}{c}{ After 60 days } \\
\cline { 2 - 9 } & N/C & GTS & Met & GTS+Met & N/C & GTS & Met & GTS+Met \\
\hline SP & $128.7 \pm 8.8$ & $132.3 \pm 6.5$ & $125.8 \pm 7.2$ & $133.1 \pm 9.6$ & $131.4 \pm 6.5$ & $126.3 \pm 8.3$ & $130.5 \pm 10.1$ & $135.2 \pm 7.7$ \\
DP & $96.5 \pm 5.6$ & $101.3 \pm 7.3$ & $91.4 \pm 8.1$ & $93.5 \pm 10.7$ & $110.4 \pm 5.8$ & $114.7 \pm 6.8$ & $112.5 \pm 9.8$ & $115.5 \pm 8.4$ \\
HR & $398.5 \pm 12.8$ & $381.4 \pm 15.8$ & $392.5 \pm 17.6$ & $388.0 \pm 19.1$ & $412.5 \pm 13.2$ & $405.3 \pm 14.2$ & $412.9 \pm 19.3$ & $403.7 \pm 15.8$ \\
PP & $32.2 \pm 7.5$ & $31.0 \pm 7.2$ & $34.4 \pm 7.7$ & $39.6 \pm 9.7$ & $21.0 \pm 6.3$ & $11.6 \pm 7.5$ & $18.0 \pm 9.5$ & $19.7 \pm 8.1$ \\
MP & $106.3 \pm 7.2$ & $111.5 \pm 6.8$ & $102.8 \pm 7.7$ & $106.7 \pm 10.3$ & $117.4 \pm 6.2$ & $118.6 \pm 7.2$ & $118.6 \pm 9.8$ & $118.8 \pm 8.2$ \\
\hline
\end{tabular}

Met; methionine, SP; systolic pressure (mmHg), DP; diastolic pressure (mmHg), HR; heart rate (beats/min), PP; pulse pressure (mmHg), $\mathrm{MP}$; mean pressure $(\mathrm{mmHg})$.

The data were obtained from groups of normal control (N/C), GTS (100 mg/kg) alone, GTS (100 mg/kg)+Met, and Met alone. Data are mean \pm S.E.M. ( $\mathrm{n}=10$, each group).

nificantly elevated after 30 and 60 days in Met alone administration group compared with the GTS+Met group; Met : GTS+Met $=42.5 \pm 5.7: 29.1 \pm 5.0 \mu M$ after 30 days and Met : GTS+Met $=$ above of $75: 43.1 \pm 9.1 \mu M$ after 60 days $\left({ }^{*} p<0.05\right.$ and ${ }^{* *} p<0.01$ significantly different from Met alone administration). Thus, co-administration of GTS reduced the mean Hcy levels by $31.6 \%$ and above of $43 \%$ after 30 and 60 days compared with Met alone group, respectively (Fig. 2). However, GTS alone administration did not affect plasma Hcy level after 30 and 60 days, indicating that GTS itself did not affect basal plasma Hcy level but GTS co-administration with Met could inhibit Met alone-caused elevation of plasma Hcy.

\section{Echocardiography in each administration group.}

In previous reports, because plasma H-Hcy can induce LV hypertrophy and cardiac fibrosis in animal models, ${ }^{16,17)}$ we examined LV-end-systolic and diastolic posterior wall thickness in each group. Systolic and diastolic cardiac LV wall thickness was measured (Fig. 3). Systolic LV-wall thickness was $2.3 \pm 0.1,2.5 \pm 0.04,3.02 \pm 0.03$ and $2.44 \pm 0.08 \mathrm{~mm}$ in normal control, GTS, Met, GTS+Met group, respectively ( $\mathrm{n}=10$, each group). Diastolic LV-wall thickness were $1.6 \pm 0.05,1.72 \pm 0.04,1.96 \pm 0.04$ and $1.72 \pm 0.04$ $\mathrm{mm}$ in normal control, GTS, Met, GTS+Met group, respectively ( $\mathrm{n}=10$, each group) (Fig. 4). Met alone group showed significant increases of LV-end-systolic and diastolic posterior wall thickness as compared with normal control group. Co-administration of GTS significantly reduced systolic cardiac and diastolic LV wall thickness of the rats as compared with Met alone group $\left({ }^{*} p<0.01\right)$.

Ginseng becomes popular in the Western world for its alleged tonic effects and possible curative and restorative properties. Accumulating evidences support the potential benefits of ginseng roots in the cardiovascular systems. For example, administration of ginsenosides, active ingredients extracted from Panax ginseng, has been shown to

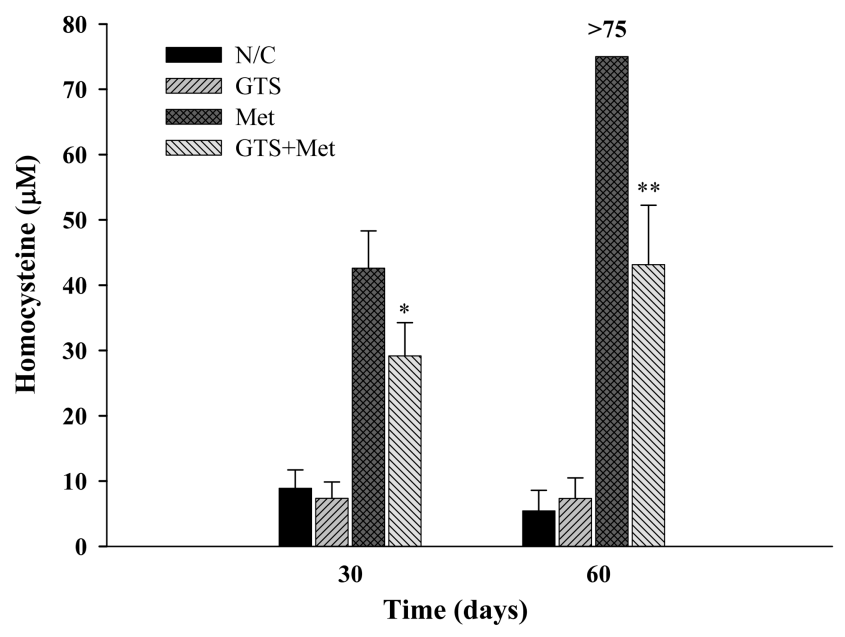

Fig. 2. Plasma total L-Hcy levels after 30 and 60 days after administration with L-methionine (Met) ( $\mathrm{n}=10$ each group). Hcy levels were significantly decreased in GTS+Met combined group as compared with Met alone group after oral administration of Met. However, this parameter was not significant different between GTS alone group and normal control. ${ }^{*} p<0.01$, vs. Met.

decrease blood pressure in both hypertensive patients and experimental animals. ${ }^{33,34)}$ The anti-hypertensive effects of ginsenosides may be at least partially due to their ability to regulate vascular tones. Indeed, ginsenosides have been shown to concentration-dependently relax the prostaglandin F2a-induced contraction of isolated rabbit pulmonary arteries ${ }^{35}$ and the phenylephrine-induced contraction of isolated rabbit rat aortas. ${ }^{34)}$ However, no previous works have examined whether GS also have effects on long-term Met-induced H-Hcy and H-Hcy-caused cardiovascular injury in rats.

The present study was performed to know whether in vivo long-term administration of GTS exhibits beneficial effects on long-term Met-induced H-Hcy and H-Hcyinduced cardiovascular damages. Our results revealed 


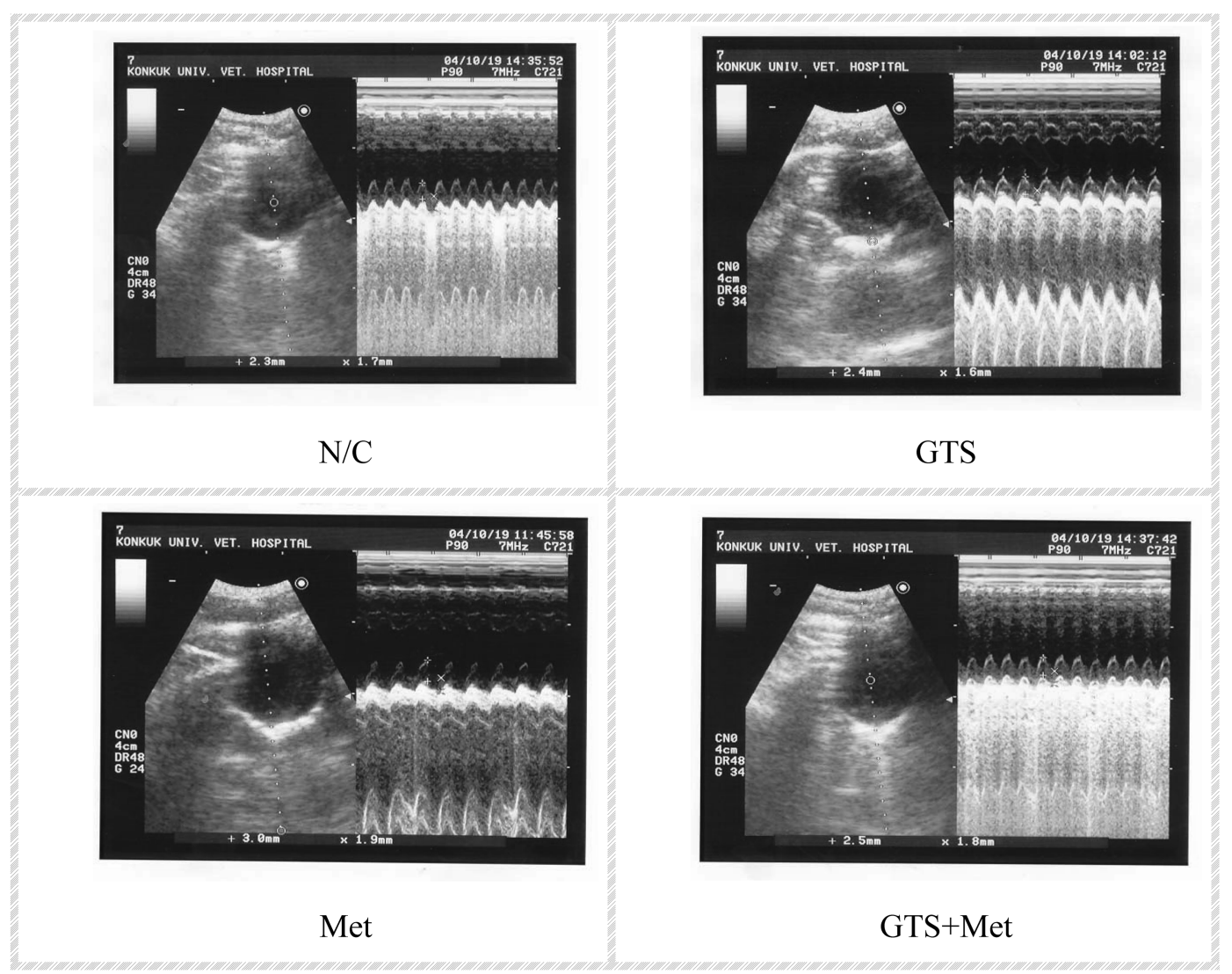

Fig. 3. Effects of GTS on cardiac remodeling and systolic and diastolic function. Representative M-mode echocardiogram at 60 days administration of Met and GS in control (Con), GTS alone, Met alone, and GTS + Met combined group.

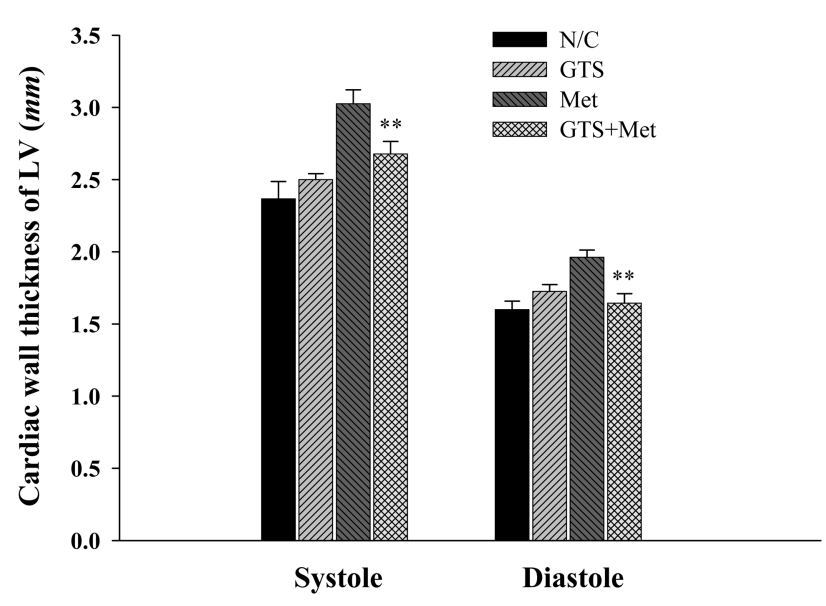

Fig. 4. Effects of GTS on cardiac remodeling and systolic and diastolic function. Serial changes of left ventricular (LV) posterior wall thickness in the LV end-systolic and diastolic pressure in each group. Values are presented as means \pm SEM. * ${ }^{*}<0.05$, vs. Met. Scale bar, $250 \mathrm{msec}$.

four major findings. First, the long-term co-administration of GTS with Met inhibited the elevation of plasma Hcy induced by long-term administration of Met (Fig. 2). Second, the long-term co-administration of GTS with Met significantly reduced Met-induced LV-end-systolic and diastolic posterior wall thickness in echocardiography (Fig. 3, 4). Thus, the major findings of the present study are that GTS has preventive effects against H-Hcy and attenuates the cardiovascular dysfunctions caused by $\mathrm{H}$ Hcy. The previous reports showed that co-administration of folate, vitamin B12, or vatamin B6 prevents the longterm administration of Met-induced H-Hcy. ${ }^{36-38)}$ The folate and vitamin 12 are involved in the remethylation of Hcy to Met, and vitamin B6 is a necessary cofactor in the degradation of Hcy to cysteine. ${ }^{39)}$ Thus, the attenuating effect of GTS against H-Hcy is unlikely due to that GTS is involved in remethylation or degradation of Met as those vitamins do. We could not currently explain how the longterm administration of GTS inhibits the elevation of plasma Hhcy induced by long-term administration of Met. There could be several speculations as adaptogenic effects of GTS. First, GTS might inhibit Met uptake in gastrointestinal systems. Second, GTS might facilitate Met 
metabolism and excretions with different ways from those of vitamins. Future studies will be required to assess these possibilities. On the other hand, NO produced from eNOS is a known regulator of vascular tone, blood pressure, and antithrombotic activity. ${ }^{40-42)}$ Thus, inadequate NO production and decreased eNOS expression due to H-Hcyinduced endothelial damages are seen in artherosclerosis and other vascular injury. ${ }^{43)}$ As mentioned above, homocysteine also increases potent reactive oxygen free radicals, and this may also explain the decreased availability of $\mathrm{NO}$, because superoxide anion is readily reacted with NO to form peroxynitrite anion. ${ }^{44)}$ Also, it is known that vascular tone is usually determined by balance between contraction and relaxation. The loss of the basal release of endothelium-derived relaxant factors by H-Hcy results in significant alterations of constriction and relaxation of vascular smooth muscle. ${ }^{37)}$ It is known that $\mathrm{H}$ Hcy also induces abnormality of heart function as well as endothelial injury. ${ }^{23)}$ In this study examining that the long-term co-administration of GTS with Met also attenuates $\mathrm{H}$-Hcy-induced heart abnormalities, we found using echocardiography that H-Hcy caused an increase of LV wall thickness, whereas co-administration of GTS with Met significantly reduced H-Hcy-induced increase of LV wall thickness. It could be then questioned what is the mechanism underlying the attenuating effect of GTS against H-Hcy-caused rat LV thickness. The primary possibility is that GTS-induced attenuation against H-Hcyinduced LV thickness might be derived from the inhibition of elevation of plasma Hcy (Fig. 2). H-Hcy may promote LV hypertrophy through vascular and non-vascular mechanisms. Hcy has growth-promoting and collagen production-stimulating effects on vascular smooth muscle cells and inhibitory effects on endothelial cell growth. ${ }^{45-47)}$ The second possibility is that GTS protection against $\mathrm{H}$ Hcy-induced LV thickness might be derived from the inhibition on Hcy-induced $\mathrm{Ca}^{2+}$ influx via $\mathrm{Ca}^{2+}$ channel on myocardial endothelium. Previous report showed that an increase in intracellular calcium increases collagen expressions in fibroblast cells. ${ }^{48)}$ Blocking of calcium channel inhibits matrix metalloproteinase expression. ${ }^{49)}$ And, the effect of Hcy in intracellular calcium release was 100 -fold greater than cysteine. ${ }^{50)}$ In the previous study, we have shown that ginsenosides inhibit L-types of $\mathrm{Ca}^{2+}$ channels. ${ }^{51-54)}$ Thus, the inhibitory effect of GTS on $\mathrm{Ca}^{2+}$ channel activity could be the basis of attenuation of $\mathrm{H}$ Hcy-induced LV thickness through inhibition of intracellular $\mathrm{Ca}^{2+}$ elevation on myocardial endothelium in rats. The third and last possibility is that the effect of GTS against LV thickness might be derived from the attenuation of oxidative stress caused by H-Hcy on myocardial endothelium as described above. ${ }^{55,56)}$ Interestingly, we could not observe significant differences in the hemodynamic parameters between normal control, GTS alone, Met alone and GTS+Met groups. Similarly, other investigators reported the unchanged systolic AP in mild H-Hcy rats as compared with control normal group. ${ }^{38,39)}$ Moreover, acute intravenous injection or infusion of high doses of Hcy to induce higher plasma Hcy levels $(65 \sim 1,000$ . I $M$ ) did not alter mean AP in the rat. ${ }^{57,58)}$ Therefore, mild H-Hcy per se has no effect on AP, at least in this species. However, the association between mild H-Hcy and higher AP observed in human studies, further studies remains to be explained. ${ }^{59-61)}$

In summary, the present study showed that the longterm administration of Met induced H-Hcy and increased wall thickness of LV-end-systolic and diastolic posterior wall thickness, whereas co-administration of GTS with Met induced an attenuation of those cardiovascular abnormalities caused by H-Hcy. These results show a possibility that GTS can be used as a prophylaxic agent attenuating $\mathrm{H}-\mathrm{Hcy}$ and H-Hcy caused-cardiovascular dysfunctions.

\section{REFERENCES}

1. Bleich S, Bandelow B, Javaheripou, K, Muller A, Degner D, Wilhelm, Finkelstein JD. Methionine metabolism in mammals: the biochemical basis for homocystinuria. Metabolism 23: 387-398 (1974)

2. Finkelstein JD. The metabolism of homocysteine: pathways and regulation. Eur J Pediatr. 157 (Suppl. 2): S40-S44 (1998)

3. Lentz SR, Sobey CG, Piegors DJ, Bhopatkar MY, Faraci FM, Malinow MR, Heistad DD. Vascular dysfunction in monkeys with diet-induced hyper- homocyst(e)inemia. J Clin Invest. 98: 24-29 (1996)

4. Ungvari Z, Pacher P, Rischak K, Szollar L, Koller A. Dysfunction of nitric oxide mediation in isolated rat arterioles with methionine diet-induced hyper- homocysteinemia. Arterioscler Thromb Vasc Biol. 19: 1899-1904 (1999)

5. Lang D, Kredan MB, Moat SJ, Hussain SA, Powell CA, Bellamy MF, Powers HJ, Lewis MJ. Homocysteine-induced inhibition of endothelium dependent relaxation in rabbit aorta role for superoxide anions. Arterioscler Thromb Vasc Biol. 20: 422-427 (2000)

6. Eberhardt RT, Forgione MA, Cap A, Leopold JA, Rudd MA, Trolliet M, Heydrick S, Stark R, Klings ES, Moldovan NI, Yaghoubi M, Goldschmidt-Clermont PJ, Farber HW, Cohen R, Loscalzo J. Endothelial dysfunction in a murine model of mild hyperhomocyst(e)inemia. J Clin Invest. 106: 483-491 
(2000)

7. Tawakol A, Omland T, Gerhard M, Wu JT, Creager MA. Hyperhomocyst(e)inemia is associated with impaired endothelium-dependent vasodilation in humans. Circulation 95: 1119-1121 (1997)

8. Bellamy MF, McDowell IF, Ramsey MW, Brownlee M, Bones C, Newcombe RG, Lewis MJ. Hyperhomocysteinemia after an oral methionine load acutely impairs endothelial function in healthy adults. Circulation 98: 1848-1852 (1998)

9. Chambers JC, McGregor A, Jean-Marie J, Obeid OA, Kooner JS. Demonstration of rapid onset vascular endothelial dysfunction after hyperhomocysteinemia: an effect reversible with vitamin C therapy. Circulation 99: 1156-1160 (1999)

10. Kanani PM, Sinkey CA, Browning RL, Allaman M, Knapp HR, Haynes WG. Role of oxidant stress in endothelial dysfunction produced by experimental hyper homocyst(e)inemia in humans. Circulation 100: 1161-1168 (1999)

11. Virdis A, Ghiadoni L, Cardinal H, Favilla S, Duranti P, Birindelli R, Magagna A, Bernini G, Salvetti G, Taddei S, Salvetti A. Mechanisms responsible for endothelial dysfunction induced by fasting hyperhomocystinemia in normotensive subjects and patients with essential hypertension. J Am Coll Cardiol. 38: 1106-1115 (2001)

12. Harker LA, Ross R, Slichter S, Scott RC. Homocystineinduced arteriosclerosis: The role of endothelial cell injury and platelet response in its genesis. J Clin Invest. 58: 731741(1976)

13. Rodgers GM, Conn MT. Homocysteine, an atherogenic stimulus, reduces protein $\mathrm{C}$ activation by arterial and venous endothelial cells. Blood 75: 895-901 (1990)

14. Welch GN, Loscalzo J. Homocysteine and atherothrombosis. N Engl J Med. 338:1042-50 (1998)

15. Fu W, Conklin BS, Lin PH, Lumsden AB, Yao Q, Chen C. Red wine prevents homocysteine induced endothelial dysfunction in porcine coronary arteries. J Surg Res. 115: 82-91 (2003)

16. Joseph J, Washington A, Joseph L et al. Hyperhomocysteinemia leads to adverse cardiac remodeling in hypertensive rats. Am J Physiol Heart Circ Physiol. 283(6): H2567-H2574 (2002)

17. Miller A, Mujumdar V, Palmer L et al. Reversal of endocardial endothelial dysfunction by folic acid in homocysteinemic hypertensive rats. Am J Hypertens. 5(2 Pt 1): 157-163 (2002)

18. Zhou W, Chai H, Lin PH, Lumsden AB, Yao Q, Chen CJ. Molecular mechanisms and clinical applications of ginseng root for cardiovascular disease. Med Sci Monit. 10: RA187RA192 (2004)

19. Gillis CN. Panax ginseng pharmacology: a nitric oxide link? Biochem Pharmacol. 54: 1-8 (1997)

20. Chan P, Thomas GN, Tomlinson B. Protective effects of trili- nolein extracted from Panax notoginseng against cardiovascular disease. Acta Pharmacol Sin. 23: 1157-1162 (2002)

21. Huang YS, Yang ZC, Yan BG, Hu XC, Li AN, Crowther RS. Improvement of early postburn cardiac function by use of Panax notoginseng and immediate total eschar excision in one operation. Burns 25: 35-41 (1999)

22. Liu JW, Wei DZ, Du CB, Zhong JJ. Enhancement of fibrinolytic activity of bovine aortic endothelial cells by ginsenoside Rb2. Acta Pharmacol Sin. 24: 102-108 (2003)

23. Sundström J, Sullivan L, Selhub J, Benjamin EJ, D'Agostino RB, Jacques PF, Rosenberg IH, Levy D, Wilson PWF and Vasan RS. Relations of plasma homocysteine to left ventricular structure and function: the Framingham Heart Study. European Heart Journal 25: 523-530 (2004)

24. Entz SR, Sobey CG, Piegors DJ, Bhopatkar MY, Faraci FM, Malinow RM, Heistad DD. Vascular dysfunction in monkeys with diet-induced hyperhomocyst(e)inemia. J. Clin. Invest. 98: 24-29 (1996)

25. Durand P, Lussier-Cacan S, Blache D. Acute methionine load-induced hyperhomocysteinemia enhances platelet aggregation, thromboxane biosynthesis, and macrophage-derived tissue factor activity in rats. FASEB J. 11: 1157-1168 (1997)

26. Durand P, Fortin LJ, Lussier-Cacan S, Davignon J, Blache D. Hyperhomocysteinemia induced by folic acid deficiency and methionine load--applications of a modified HPLC method. Clin. Chim. Acta, 252: 83-93 (1996)

27. Lentz SR, Sobey CG, Piegors DJ, Bhopatkar MY, Faraci FM, Malinow RM, Heistad DD. Vascular dysfunction in monkeys with diet-induced hyperhomocyst(e)inemia. J Clin Invest. 98: 24-29 (1996)

28. Durand P, Lussier-Cacan S, Blache D. Acute methionine load-induced hyperhomocysteinemia enhances platelet aggregation, thromboxane biosynthesis, and macrophage-derived tissue factor activity in rats. FASEB J. 11: 1157-1168 (1997)

29. Durand P, Fortin LJ, Lussier-Cacan S, Davignon J, Blache D. Hyper- homocysteinemia induced by folic acid deficiency and methionine load: applications of a modified HPLC method. Clin Chim Acta. 252: 83-93 (1996)

30. Ulbink JB, Vermak WJH, Bissbort S. Rapid high-performance liquid chromatographic assay for total homocysteine levels in humans serum. J Chromatogr B Biomed Appl. 565: 441-446 (1991)

31. Miao CY, Su DF. The importance of blood pressure variability in rat aortic and left ventricular hypertrophy produced by sinoaortic denervation. J Hypertens. 20: 1865-1872 (2002)

32. Litwin SE, Katz SE, Morgan JP, Douglas PS. Serial echocardiographic assessment of left ventricular geometry and function after large myocardial infarction in the rat. Circulation 89: 345-354 (1994)

33. Sohn ES, Huh BY, Park SC, Park CW, Kim HJ. The effect of ginseng on blood pressure in spontaneous hypertensive rat 
and essential hypertension. Proceedings of the 3rd International Ginseng Symposium. Korean Ginseng Research Institute Seoul, Korea. 1-3 (1980)

34. Kim ND, Kang SY, Schini VB. Ginsenosides evoke endothelium-dependent vascular relaxation in rat aorta. Gen. Pharmacol. 25: 1071-1077 (1994)

35. Chen X, Gillis CN, Moalli R. Vascular effects of ginsenosides in vitro. Br. J. Pharmacol. 82: 485-491 (1984)

36. Lentz SR, Sobey CG, Piegors DJ, Bhopatkar MY, Faraci FM, Malinow MR, Heistad DD. Vascular dysfunction in monkeys with dietinduced hyper- homocyst(e)inemia. J Clin Invest. 98: 24-29 (1996)

37. Ungvari Z, Pacher P, Rischak K, Szollar L, Koller A. Dysfunction of nitric oxide mediation in isolated rat arterioles with methionine diet-induced hyper-homocysteinemia. Arterioscler Thromb Vasc Biol. 19: 1899-1904 (1999)

38. Morita H, Saito Y, Kurabayashi M, Nagai R. Diet-induced mild hyper- homocysteinemia and increased salt intake diminish vascular endothelial function in a synergistic manner. J Hypertens. 20: 55-62 (2002)

39. De Bree A, Verschuren WM, Kromhout D, Kluijtmans LAJ, Blom HJ. Homocysteine determinants and the evidence to what extent homocysteine determines the risk of coronary heart disease. Pharmacol Rev. 54: 599-618 (2002)

40. Patel RP, McAndrew J, Sellab H, White CR, Jo H, Freeman $\mathrm{BA}$, et al. Biological aspects of reactive nitrogen species. Biochem Biophys Acta 1411: 385-400 (1999)

41. Radomski MW, Moncada S. Regulation of vascular homeostasis by nitric oxide. Thromb Haemost. 70: 36-41 (1993)

42. Lamas S, Perez-Sala D, Moncada S. Nitric oxide: from discovery to the clinic. Trends Pharmacol Sci. 19: 436-438 (1998)

43. Channon KM, Blazing MA, Shetty GA, Potts KE, George SE. Adenoviral gene transfer of nitric oxide synthase: high level expression in human vascular cells. Cardiovasc Res. 32: 962-972 (1996)

44. Beckman JS, Koppenol WH. Nitric oxide, superoxide, and peroxynitrite: the good, the bad, and the ugly. Am J Physiol. 271: C1424-1437 (1996)

45. Majors A, Ehrhart LA, Pezacka EH. Homocysteine as a risk factor for vascular disease: enhanced collagen production and accumulation by smooth muscle cells. Arterioscler Thromb Vasc Biol. 17(10): 2074-2081 (1997)

46. Tsai JC, Perrella MA, Yoshizumi M et al. Promotion of vascular smooth muscle cell growth by homocysteine: a link to atherosclerosis. Proc Natl Acad Sci USA 91(14): 6369-6373 (1994)

47. Tyagi SC. Homocysteine redox receptor and regulation of extracellular matrix components in vascular cells. Am J Physiol. 274(2 Pt 1): C396-405 (1998)
48. Hori M, Sato H, Fukunami M, Houki N. Pathophysiology of cytoskeleton and ECM in failing myocardium. Jpn J Clin Med. 51: 1191-1197 (1993)

49. Kohn EC, Jacobs W, Kim YS, Alessandro R, Stetler-Stevenson WG, Liotta LA. Calcium influx modulates expression of matrix metalloproteinase-2 (72-kDa type IV collagenase, gelatinase A). J Biol Chem. 269(21): 505-21511 (1994)

50. Mujumdar VS, Hayden MR, Tyagi SC. Homocyst(e)ine Induces Calcium Second Messenger in Vascular Smooth Muscle Cells. Journal of Cellular Physiology 183: 28-36 (2000)

51. Nah SY, McCleskey EW. Ginseng root extract inhibits calcium channels in rat sensory neurons through a similar path, but di.erent receptor, as -type opioids. J Ethnopharmacol. 42: 45-51 (1994)

52. Nah SY, Park HJ, McCleskey EW. A trace component of ginseng that inhibits $\mathrm{Ca}^{2+}$ channels through a pertussis toxinsensitive G protein. Proc Natl Acad Sci USA 92: 8739-8743 (1995)

53. Choi S, Jung SY, Kim CH, Kim HS, Rhim H, Kim SC, Nah SY. Effect of ginsenosides on voltage-dependent $\mathrm{Ca}^{2+}$ channel subtypes in bovine chromafin cells. J Ethnopharmacol. 74: 75-81 (2001)

54. Rhim H, Kim H, Lee DY, Oh TH, Nah SY. Ginseng and ginsenoside $\mathrm{Rg}_{3}$, a newly identified active ingredient of ginseng, modulate $\mathrm{Ca}^{2+}$ currents in rat sensory neurons. Eur $\mathrm{J}$ Pharmacol. 436: 151-158 (2002)

55. Chambers JC, McGregor A, Jean-Marie J et al. Acute hyperhomocysteinaemia and endothelial dysfunction. Lancet 351(9095): 36-37 (1998)

56. Lentz SR, Sobey CG, Piegors DJ et al. Vascular dysfunction in monkeys with diet-induced hyperhomocyst(e)inemia. J Clin Invest. 98(1): 24-29 (1996)

57. Dudman NP, Temple SE, Guo XW, Fu W, Perry MA: Homocysteine enhances neutrophilendothelial interactions in both cultured human cells and rats in vivo. Circ Res. 84 :409-416 (1999)

58. Fu WY, Dudman NP, Perry MA, Wang XL. Leukocytes extravasation in acute homocysteinemic rats. Atherosclerosis 161: 177-183 (2002)

59. Van Guldener C, Nanayakkara PW, Stehouwer CD. Homocysteine and blood pressure. Curr Hypertens Rep. 5: 26-31 (2003)

60. Nygard O, Vollset SE, Refsum H, Stensvold I, Tverdal A, Nordrehaug JE, Ueland M, Kvale G. Total plasma homocysteine and cardiovascular risk profile. The Hordal and Homocysteine Study. JAMA. 274: 1526-1533 (1995)

61. Lim U, Cassano PA. Homocysteine and blood pressure in the Third National Health and Nutrition Examination Survey, 1988-1994. Am J Epidemiol. 156: 1105-1113 (2002) 